\title{
A NEW MEASURE OF GROWTH FOR COUNTABLE-DIMENSIONAL ALGEBRAS
}

\author{
JOHN HANNAH AND K. C. O'MEARA
}

\begin{abstract}
A new dimension function on countable-dimensional algebras (over a field) is described. Its dimension values for finitely generated algebras exactly fill the unit interval $[0,1]$. Since the free algebra on two generators turns out to have dimension 0 (although conceivably some Noetherian algebras might have positive dimension!), this dimension function promises to distinguish among algebras of infinite GK-dimension.
\end{abstract}

\section{INTRODUCTION}

In recent times, the most prominent dimension used in the study of algebras has been the Gelfand-Kirillov dimension (GK-dimension), which measures the "growth of an algebra in terms of generators" (see [2]). Here we present another view of "growth of an algebra", based on certain infinite matrix representations. By an algebra we shall always mean an associative algebra over a field, with an identity element.

It would be unthinkable that one could have a serious study of finite-dimensional algebras without ever resorting to finite matrix representations. In the theory of infinite-dimensional algebras, however, infinite matrix representations have played only a very minor role. One reason for this, perhaps, is that the nice "arithmetic" functions provided by a finite matrix representation-such as trace, determinant, rank, etc. - would appear to have no cousins in the infinitedimensional case. However, a recent and surprising result by Goodearl, Menal, and Moncasi [1, Proposition 2.1] offers fresh hope for infinite matrix representations of countable-dimensional algebras $A$ over a field $F$ : it says that such $A$ can be embedded in the algebra $B(F)$ of all $\omega \times \omega$ matrices over $F$ which are simultaneously row-finite and column-finite. (Note $\omega \times \omega$ matrices are just $\aleph_{0} \times \aleph_{0}$ matrices with their rows and columns ordered in the standard way.) This result has been the inspiration for our work. For in any such representation of $A$, the elements of $A$ now have all their nonzero entries relatively close to the main diagonal. This raises the question of just how closely these nonzero entries can be squeezed to the main diagonal for a suitable embedding. To help quantify this, we introduce the notion of a growth curve for an element $x \in B(F)$. We say that a function $g: \mathbf{N} \rightarrow \mathbf{R}^{+}$is a growth curve for $x \in B(F)$ if, for each $n \in \mathbf{N}, x(n, i)=0=x(i, n)$ for all $i>n+g(n)$. In other words, $g(n)$ gives a bound on the "bandwidth" of $x$ at the $(n, n)$ position, if we interpret bandwidth as in Figure 1. (There are other interpretations of "bandwidth", of course.)

We say that $x \in B(F)$ has at most order $g(n)$ growth (or that $x$ has $\mathrm{O}(g(n)$ )

Received by the editors October 22, 1992.

1991 Mathematics Subject Classification. Primary 16P90, 16S50; Secondary 16S15, 16 G99. 


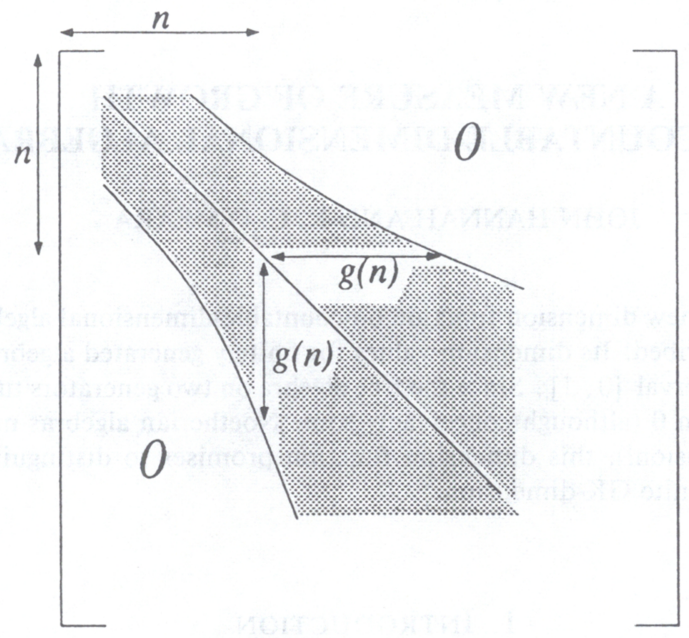

FIGURE 1

growth) if there is a constant $c>0$ such that the function $c g(n)$ is a growth curve for $x$. If $A$ is a subalgebra of $B(F)$ and every $x \in A$ has $\mathrm{O}(g(n))$ growth, then we say that the algebra $A$ itself has $\mathrm{O}(g(n))$ growth (but notice that the constant $c$ in $c g(n)$ will depend on the particular $x \in A)$. To say $A$ has linear growth means $A$ has $\mathrm{O}(n)$ growth.

\section{Statement of main Results}

Our first theorem is an improvement on the Goodearl, Menal, and Moncasi embedding mentioned earlier.

Theorem 1. Every countable-dimensional algebra $A$ over a field $F$ can be embedded in $B(F)$ as a subalgebra of linear growth.

The proof relies on two key results. The first says that for any finite number $k$ of linear transformations of a countable-dimensional vector space, there is a simultaneous block tridiagonal matrix form of them in which the sizes of the diagonal blocks are

$$
1,2 k+1,(2 k+1)^{2}, \ldots,(2 k+1)^{n}, \ldots .
$$

The second result is the fact that any countable-dimensional algebra can be embedded in some finitely generated algebra [3]. We stress that all our algebra embeddings are required to preserve the identity. Full details of the proof will appear elsewhere.

Can we improve on Theorem 1? The answer is "no" in general, or at least not in terms of $\mathrm{O}\left(n^{r}\right)$ growth for any $r<1$. But there is an interesting range of "sublinear" growths which we can identify for individual algebras. For each real number $r \in[0,1]$ let

$$
G(r)=\left\{x \in B(F) \mid x \text { has } \mathrm{O}\left(n^{r}\right) \text { growth }\right\} .
$$

These $G(r)$ are subalgebras of $B(F)$, and Theorem 1 says each countabledimensional algebra $A$ can be embedded in $G(1)$. We take the "least" $r$ for which $A$ embeds in $G(r)$ as our new dimension for $A$. 
Definition. The bandwidth dimension of a countable-dimensional algebra $A$ (over a field $F$ ) is

$$
\inf \{r \in \mathbf{R}, r \geq 0 \mid A \text { embeds in } G(r)\}
$$

or, equivalently,

$$
\inf \left\{r \in \mathbf{R}, r \geq 0 \mid A \text { embeds in } B(F) \text { with } \mathrm{O}\left(n^{r}\right) \text { growth }\right\} .
$$

Our second theorem completely describes the possible range of values of our dimension function on finitely generated algebras and, hence, also on countabledimensional algebras (by Theorem 1). It suggests that bandwidth dimension is somewhat "smoother" than $G K$-dimension, whose corresponding range of values on finitely generated algebras is

$$
0,1,2 \text {, any real } r>2 \text {, and }+\infty \text {. }
$$

Theorem 2. For any field $F$, bandwidth dimensions of finitely generated algebras over $F$ exactly fill the unit interval $[0,1]$.

The proof (over 20 pages) will appear elsewhere in a paper by the second author. A sketch is provided later.

It is not difficult to show that the free algebra $F\{x, y\}$ on two generators embeds in the algebra $G(0)$ of finite bandwidth matrices and so has zero growth. Accordingly, its bandwidth dimension is the smallest possible value, namely, 0 . In sharp contrast, from the $G K$ point of view this algebra has exponential growth and, therefore, the largest possible GK-dimension, namely, $+\infty$. This suggests that bandwidth dimension is quite different from $G K$-dimension.

Bandwidth dimension behaves as one would hope with respect to subalgebras, finite subdirect products, and finite matrix algebras. For instance, an algebra $A$ and its matrix algebra $M_{n}(A)$ have the same dimension. However, bandwidth dimension behaves poorly on factor algebras-but this is the expected price we pay for not giving the free algebra the largest possible dimension.

There are good signs that certain bandwidth dimension values may be reflected in interesting purely ring-theoretic properties. As an illustration of how a growth curve restriction can result in quite strong ring properties, we quote:

Proposition. Suppose $R$ is a (von Neumann) regular, right self-injective ring.

(1) If $R$ has linear growth over some field $F$ (i.e., $R$ embeds in $G(1)$ ), then $R$ must be of Type $I_{f}$.

(2) If $R$ has zero growth (i.e., $R$ embeds in $G(0)$ ), then $R$ must have bounded index of nilpotence.

\section{SKetch OF THE PROOF OF THEOREM 2}

Let $r \in[0,1]$. We shall construct a finitely generated algebra $A$ of bandwidth dimension $r$. The case $r=0$ is trivial, and the case $r=1$ turns out to be taken care of by any "purely infinite" $A$, that is, $A \cong A \oplus A$ as right $A$ modules. Therefore, we can assume $0<r<1$. Let $t=r /(1-r)$, and for each $k \in \mathbf{N}$ let $n_{k}=\left[k^{t}\right]$ where [ ] denotes the integer part. In order to absorb some of the flavor of the proof, consider first the natural copy $R$ of $\prod_{k=1}^{\infty} M_{n_{k}}(F)$ inside $B(F)$, that is, $R$ consists of all the block-diagonal matrices of the form shown in Figure 2. Let $J_{k}$ be the $k$ th diagonal block for each $k \in \mathbf{N}$ (so 


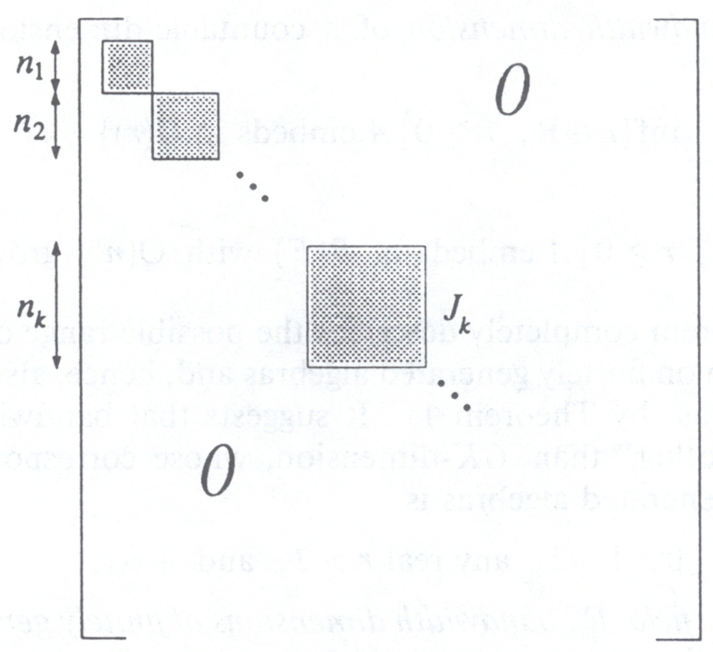

FIGURE 2

$\left.J_{k} \cong M_{n_{k}}(F)\right)$. The $n_{k}$ have been designed so that $R \subseteq G(r)$ but $R \nsubseteq G(s)$ for any $s<r$. Even though $R$ is not countable dimensional, we can still talk of its bandwidth dimension (as an abstract algebra). Although at first sight this looks like it ought to be $r$, in fact the bandwidth dimension is 0 because $R$ can be embedded in $G(s)$ for any $0<s<r$, simply by "stretching" out the above representation and repeating blocks often enough. Preventing this stretching is one of the key problems addressed in the proof. In the case of $R$, it turns out that the algebra $B$ generated by $R$ and the standard one-dimensional infinite shift is sufficiently "rigid" to have bandwidth dimension exactly $r$. But, of course, $B$ is far from being finitely generated!

In the proof of Theorem 2, we construct $A$ as an 8-generator subalgebra of $G(r)$ with the following:

Key Property. A contains each $J_{k}$, and the number of products of generators needed to obtain each of the standard matrix units in $J_{k}$ grows essentially logarithmically in $k$; in fact, we can get by with $\mathrm{O}\left((\log k)^{2}\right)$ products.

This property is achieved by including various infinite shift matrices among the generators of $A$ and also a block diagonal matrix of strategically placed $2^{m} \times 2^{m}$ "binary search" matrices for various $m$. For elements $a, b$ of a general ring $T$, we say $\gamma \in T$ is a cross-element from $b T$ to $a T$ if $\gamma T=a T$ and $T \gamma=T b$ (herein lies the significance for us of matrix units). By including the standard one-dimensional infinite shift among our generators, we can ensure that $A$ contains a cross-element from any standard primitive idempotent of $J_{k}$ to any one of $J_{k+1}$, also in $\mathrm{O}\left((\log k)^{2}\right)$ products of generators.

Now suppose $\theta: A \rightarrow G(s)$ is an algebra embedding for some $s<1$. To complete the proof, we show, in several steps, that $r \leq s$. For each $c \in \mathbf{R}^{+}$let

$$
W_{s}(c)=\left\{x \in B(F) \mid c n^{s} \text { is a growth curve for } x\right\} .
$$

Notice that $G(s)$ is the union of these subspaces $W_{s}(c)$. Because $s<1$, the powers of $W_{s}(c)$ grow "polynomially". 
Step 1. For any $c \in \mathbf{R}^{+}$, there exists $d \in \mathbf{R}^{+}$such that for all $m \in \mathbf{N}$

$$
\left(W_{s}(c)\right)^{m} \subseteq W_{s}\left(d m^{1 / 1-s}\right) .
$$

(We remark that for $s=0$, the $W_{s}(c)$ actually give a filtering of $G(0)$. At the other extreme, for $s=1$ the powers of $W_{s}(c)$ grow exponentially.)

For each $k \in \mathbf{N}$, fix a set $F_{k}$ of $n_{k}$ equivalent orthogonal nonzero idempotents of $J_{k}$ (for example, the standard primitives). From the above key property and Step 1, we obtain:

Step 2. There exist nondecreasing $c_{k} \in \mathbf{R}^{+}$for $k=1,2,3 \ldots$ such that

(1) $c_{k} n^{s}$ is a growth curve for elements in $\theta\left(F_{k}\right)$, for a cross-element between each pair, and for a cross-element between any element of $\theta\left(F_{k}\right)$ and one of $\theta\left(F_{k+1}\right)$.

(2) $c_{k}=\mathrm{O}\left((\log k)^{2 /(1-s)}\right)$.

Our strategy now is to chase the first nonzero rows of the images of the idempotents in $F_{k}$ and obtain opposing constraints on their positions. On the one hand, they have to be fairly close together to ensure that some cross-element from $\theta\left(F_{k}\right)$ to $\theta\left(F_{k+1}\right)$ can lie in $W_{s}\left(c_{k}\right)$. On the other hand, having the $n_{k}$ images of the equivalent orthogonal idempotents from $F_{k}$ all inside $W_{s}\left(c_{k}\right)$ forces their nonzero rows to become increasingly scattered. A comparison of these opposing growths leads to:

Step 3. $n_{k}=\mathrm{O}\left(k^{s / 1-s} c_{k}^{(1+s) /(1-s)}\right)$.

From (2) of Step 2, this yields $n_{k}=\mathrm{O}\left(k^{s / 1-s}(\log k)^{u}\right)$ where $u=$ $2(1+s) /(1-s)^{2}$. But by definition, $n_{k}$ has polynomial growth of $k^{t}$. Hence $t \leq s /(1-s)$, which implies $r /(1-r) \leq s /(1-s)$ and so $r \leq s$, as required.

\section{REFERENCES}

1. K. R. Goodearl, P. Menal, and J. Moncasi, Free and residually artinian regular rings, J. Algebra 156 (1993), 407-432.

2. G. R. Krause and T. H. Lenagan, Growth of algebras and Gelfand-Kirillov dimension, Pitman, New York, 1985.

3. K. C. O'Meara, C. I. Vinsonhaler, and W. J. Wickless, Identity-preserving embeddings of countable rings into 2-generator rings, Rocky Mountain J. Math. 19 (1989), 1095-1105.

Department of Mathematics, University of Canterbury, Christchurch 1 , New ZEALAND

E-mail address: jjh@math.canterbury.ac.nz

E-mail address: kco@math.canterbury.ac.nz 